Relations industrielles

Industrial Relations

Moving Up in the New Economy: Career Ladders for U.S. Workers, par Joan FITZGERALD, Ithaca, N.Y.: Cornell University Press, 2006, 251 p., ISBN: 0-8014-4413-6

\title{
Catherine Beaudry
}

Volume 62, numéro 2, printemps 2007

URI : https://id.erudit.org/iderudit/016094ar

DOI : https://doi.org/10.7202/016094ar

Aller au sommaire du numéro

\section{Éditeur(s)}

Département des relations industrielles de l'Université Laval

\section{ISSN}

0034-379X (imprimé)

1703-8138 (numérique)

Découvrir la revue

Citer ce compte rendu

Beaudry, C. (2007). Compte rendu de [Moving Up in the New Economy: Career Ladders for U.S. Workers, par Joan FITZGERALD, Ithaca, N.Y.: Cornell University Press, 2006, 251 p., ISBN: 0-8014-4413-6]. Relations industrielles / Industrial Relations, 62(2), 379-381. https://doi.org/10.7202/016094ar

Tous droits réservés @ Département des relations industrielles de l'Université Laval, 2007
Ce document est protégé par la loi sur le droit d'auteur. L'utilisation des services d'Érudit (y compris la reproduction) est assujettie à sa politique d'utilisation que vous pouvez consulter en ligne.

https://apropos.erudit.org/fr/usagers/politique-dutilisation/ 
general, equal work, and equal valueand the specific policy responses appropriate to each. Gender-based wage discrimination has received considerably more policy attention than race-based discrimination in all the countries discussed. In comparing the six countries, Weiner notes that Canada has the most highly developed policy framework to address pay discrimination against women. Data deficiencies hinder efforts to compare outcomes of the policy approaches in different countries, though there is evidence that fair wage policies are effective in addressing pay inequality within a country.

The book is a collection of separate chapters that are not integrated by means of an analytical or conceptual framework that provides a systematic comparison of policies and results across the six countries. The theoretical chapter does not provide such a framework, nor does it go beyond traditional and outdated economic models to include more recent perspectives that address systemic discrimination. The reader is left without theoretical guidance for understanding affirmative action and employment equity policies as responses to structural and institutional discrimination.

However, an underlying theme of the book is that proactive, legislated and mandatory policy responses to employment discrimination are more effective in getting results than complaint-based systems that require individual victims of discrimination to seek justice. Another theme is the question of whether there is global convergence among countries in equality policies and practices. The conclusion notes significant variation among the countries, rooted in their local histories and institutional frameworks, that makes convergence unlikely. This is but one question of many that deserve attention in future comparative research on equality policies and their results.

Carol Agocs University of Western Ontario

\section{Moving Up in the New Economy: Career Ladders for U.S. Workers,} par Joan FITZGerald, Ithaca, N.Y.: Cornell University Press, 2006, 251 p., ISBN: 0-8014-4413-6

De toutes parts, tant dans la littérature scientifique que dans les ouvrages destinés au grand public, un nombre important d'auteurs constatent l'émergence d'un nouveau modèle de développement économique. Joan Fitzgerald s'inscrit dans ce courant et présente un ouvrage sur la trajectoire de carrière des travailleurs dans le cadre de cette «nouvelle économie ». L'auteure s'intéresse plus particulièrement aux liens entre les tendances économiques actuelles, l'éducation et le développement de la main-d'œuvre.

Précisément, Fitzgerald soulève trois tendances propres à la nouvelle économie susceptibles d'influencer la trajectoire de carrière des travailleurs. La première tendance observée par l'auteure relève du déclin du syndicalisme. Elle fait également état des pressions subies par les entreprises pour réduire leur coût de fonctionnement, notamment les coûts liés à la main-d'œuvre, et cela à cause de l'accroissement de la concurrence au cours des dernières années. Finalement, Fitzgerald aborde une dernière tendance, soit la transition d'une économie industrielle vers une économie de services. Selon l'auteure, ces trois tendances font en sorte que la nouvelle économie s'avère plus propice à la disparité salariale, offre moins de possibilités d'avancement pour les travailleurs et fragilise leur sécurité d'emploi.

Dans ce contexte, Fitzgerald propose de soutenir les travailleurs, tant au plan 
salarial qu'au plan de la mobilité, à l'aide de programmes de développement de carrières. Ces programmes visent en fait à faciliter l'apprentissage de nouvelles connaissances et l'accès à des emplois mieux rémunérés et plus qualifiés. Ils s'adressent généralement aux travailleurs qui se situent au bas de l'échelle salariale.

Moving Up in the New Economy s'attarde donc à la progression des travailleurs américains dans les organisations. C'est ainsi que l'auteure propose, au sein du premier chapitre, trois questions de départ qui structureront le livre entier. Elle cherche en premier lieu à savoir ce qui fait le succès des programmes de développement de carrière. Elle cherche également à connaître l'impact réel de ces programmes sur les travailleurs. Finalement, elle s'interroge sur le rôle du gouvernement et sur sa capacité à promouvoir le développement de la carrière des Américains.

En s'appuyant sur ces trois interrogations, Fitzgerald explore les avantages et les limites de programmes déjà en place aux États-Unis. C'est ainsi que, dans chacun des chapitres, un secteur spécifique de l'économie est étudié et que divers cas sont présentés. L'accent est mis, pour chacun des programmes, sur la façon dont il a été implanté, sur l'influence d'intermédiaires (syndicats, fondations, organismes communautaires, etc.) et sur l'appropriation par les partenaires de la législation et des politiques publiques en place.

Les trois premiers secteurs étudiés font partie de l'industrie des services aux personnes. Le chapitre 2 traite ainsi plus spécifiquement des occupations liées à la santé. Fitzgerald y présente divers programmes qui font appel à trois stratégies : l'augmentation des salaires et l'amélioration des emplois existants; la création de catégories au sein des occupations et la possibilité d'avancer vers des occupations mieux rémunérées et requérant une plus grande qualification. Suite à l'analyse de ces trois stratégies, l'auteure fait ressortir les principaux facteurs de succès des programmes. À cet égard, elle indique l'importance de faciliter la communication entre les divers niveaux hiérarchiques, d'offrir du support aux adultes qui participent aux programmes et d'établir un partenariat qui bénéficie à tous les groupes impliqués dans le programme. Elle souligne aussi la nécessité que les employeurs et que les artisans du programme soient dévoués, que les programmes soient réellement reconnus par les employeurs et que leurs exigences soient présentées aux travailleurs. Dans le même ordre d'idées, Fitzgerald explore, au chapitre 3 , les programmes de carrière mis sur pied pour les travailleurs qui s'occupent d'enfants d'âge préscolaire. Les stratégies qui y sont déployées sont semblables à celles des programmes du secteur de la santé. Suite à l'implantation des programmes en question, l'auteure remarque qu'en plus de faciliter l'accès à de meilleurs emplois, les actions entreprises permettent également d'améliorer la qualité des services offerts. Fitzgerald termine l'étude de cette industrie, au chapitre 4, par l'analyse des programmes spécifiques au secteur de l'éducation. Elle s'attarde plus spécifiquement au cas des enseignantsassistants qui souhaiteraient obtenir des diplômes d'enseignement. Elle indique que le succès de ces programmes repose généralement sur leur flexibilité, de façon à faciliter la conciliation travailétude-famille, sur le support que procure la communauté aux travailleurs, sur une bonne sélection des candidats et sur la stabilité des fonds voués aux programmes.

Les deux chapitres qui suivent s'attardent à l'industrie manufacturière. Fitzgerald se concentre d'abord, au chapitre 5, sur le secteur effervescent des biotechnologies. Or, ce secteur est particulier puisqu'il fait appel à des 
travailleurs hautement qualifiés qui ont la capacité de s'adapter aux besoins changeants des entreprises. De ce fait, les travailleurs doivent avoir régulièrement accès à divers types de formation et sont appelés à réintégrer plusieurs fois les programmes au cours de leur carrière. Dans ce contexte, les programmes étudiés par Fitzgerald encouragent généralement les travailleurs à poursuivre leur formation mais n'offrent que très peu de garanties quant aux possibilités d'avancement et quant aux augmentations salariales. De plus, les travailleurs qui au départ ne possèdent que de très faibles qualifications éprouvent le plus souvent de la difficulté à intégrer ce secteur d'activité, puisque des connaissances scientifiques et des habiletés mathématiques sont essentielles pour y exercer une fonction. Outre les biotechnologies, l'industrie manufacturière est également caractérisée par des secteurs plus traditionnels, comme la métallurgie ou le secteur agroalimentaire. La compétition y est forte. Les employeurs, pour y faire face, ont bien souvent une stratégie de réduction des coûts. Fitzgerald propose une autre voie au chapitre 6 , celle de l'amélioration de la productivité et de la qualité par l'adoption de méthodes plus avancées et, par le fait même, par le recours aux qualifications des travailleurs. Dans ce contexte, elle indique que les meilleures initiatives sont celles qui regroupent une multiplicité de partenaires (les employeurs, les syndicats, les collèges, etc.).
Finalement, le dernier chapitre se veut une synthèse des questions traitées dans l'ouvrage. Fitzgerald reprend donc les facteurs de succès des programmes et se penche sur les types de politiques qui supportent les stratégies de développement de carrière et de progression salariale.

Avec Moving $U p$ in the New Economy, Fitzgerald aborde une voie nouvelle et positive pour les travailleurs dans un contexte d'incertitudes et de précarité. La réflexion proposée par l'auteure s'avère intéressante, en ce sens où l'ouvrage fait ressortir non seulement l'importance de la volonté des travailleurs pour leur mobilité mais également l'importance de l'implication des employeurs, de celle des actions gouvernementales en matière d'emploi et de celle de la communauté. L'ensemble des acteurs de monde du travail y est donc pris en compte. Ainsi, la responsabilité de la trajectoire de carrière des travailleurs n'est pas individuelle, comme le suppose le concept fort populaire d'employabilité mis de l'avant par certains économistes du travail, mais doit plutôt être prise en charge par toute la collectivité. Fitzgerald propose donc une réflexion originale, en ce qu'elle fait contrepoids aux tendances actuelles visant la dérégulation des marchés du travail.

\section{Catherine Beaudry} Université Laval

\section{Leviathans : Multinational Corporations and the New Global History,} sous la direction d'Alfred D. CHANDLER, Jr. et Bruce MAZLISH, Cambridge : Cambridge University Press, 2005, 264 p., ISBN : 0-5215-4993-0

Alors qu'au cours de la dernière décennie, plusieurs ouvrages tentant d'élucider la problématique autour de la gestion, des structures, des stratégies et de l'impact des entreprises multinationales ont vu le jour, ce collectif réussit à se distinguer par son originalité et susciterait autant l'intérêt des chercheurs avertis que des lecteurs qui voudraient en savoir un peu plus sur le sujet. Écrit sous la direction d'Alfred Chandler, Jr. et de Bruce Mazlish, cet 